\title{
Microorganism Nutrition Processes as a General Route for the Preparation of Bionic Nanocomposites Based on Intractable Polymers
}

\author{
L. Valentini, ${ }^{*} \dagger$ S. Bittolo Bon, ${ }^{\dagger, \ddagger}$ and N. M. Pugno ${ }^{*},, \S, \perp$ \\ † Dipartimento di Ingegneria Civile e Ambientale, Universitàdi Perugia, UdR INSTM, Strada di Pentima 4, 05100 Terni, \\ Italy \\ ‡ Laboratory of Bio-Inspired and Graphene Nanomechanics, Department of Civil, Environmental and Mechanical \\ Engineering, University of Trento, via Mesiano 77, I-38123 Trento, Italy \\ $\S$ Centre for Materials and Microsystems, Fondazione Bruno Kessler, via Sommarive 18, I-38123 Povo (Trento), Italy \\ $\perp$ School of Engineering and Materials Science, Queen Mary University of London, Mile End Road, E1 4NS London, \\ United Kingdom
}

\begin{abstract}
In this paper the fermentation process activated by living microorganisms of the baker's yeast is proposed as a facile assembly method of hybrid nanoparticles at liquid interface. Water dispersion of commercial baker's yeast extract used for bread production, graphene nanoplatelets (GNPs), and carbon nanotubes (CNTs) were added to oil/water interface; when the yeast is activated by adding sugar, the byproduct carbon dioxide bubbles migrate from the water phase to the oil/water interface generating a floating nanostructured film at liquid interface where it is trapped. Starting from this simple method, we propose a general approach for the stabilization of intractable poly(etheretherketone) polymeric particles with GNPs and CNTs at immiscible liquid interface. This process allowed the formation of sintered porous composites with improved mechanical properties. The porous structure of the composites gave rise to a low thermal conductivity making them good candidates for thermal insulating applications. Liquid absorption by these porous composites has been also reported. We believe that this new approach may have applications in the large scale fabrication of nanomaterials and is particularly suited for the preparation of nanocomposites starting from polymers that are intractable by solvent casting.
\end{abstract}

\section{Keywords}

graphene, carbon nanotubes, porous nanocomposites, hybrid materials, biogenic materials

\section{Introduction}

The excellent properties of nanostructures with controlled geometry opened novel opportunities for designing materials with new functionalities; thus in view of this added value for different applications, the hierarchical assembly of nanostructures was deeply investigated.1-3 Liquid/liquid interfaces have been considered for self-assembly of many materials and in particular of graphene taking the key advantage, like in the case of water/oil interface, of the high interfacial tension.4-8 Kim et al.,4 for example, exploited the amphiphilic graphene oxide characteristics to investigate its activity at air-water, liquid- liquid, and liquid-solid interfaces. High quality graphene has been prepared by solvothermal treatment. By introducing cyclohexane and water, graphene was successfully separated and a graphene film was formed by controlling the volume of graphene/solvent solution.

It is also well-known that the partial wetting of the polymeric particles by two immiscible liquids stabilizes particles at the water-oil interface during emulsification, leading to stable water-in-oil emulsions.9-11 The particle-stabilized emulsions can be processed into highly porous solid polymer components upon drying and sintering. Some polymers, such as poly- (vinylidene difluoride) (PVDF), 
poly(tetrafluoroethylene) (PTFE), and poly(etheretherketone) (PEEK), that are intractable by solvent casting due to their insolubility in most polar solvents were successfully processed by emulsion method.9-11

The liquid-liquid interface mimics the self-assembly process occurring in nature in biological systems (e.g., microbes, fungi, plants etc.) where the metabolic activities of microorganisms lead to the formation of hierarchical structures with unique properties.12-14 For example we found that a solution of graphene, baker's yeast, and sugar can be mixed with water providing added nutrients for microorganisms resulting in the formation of hierarchical nanotemplate with novel outstanding properties.15 Moreover, it was demonstrated that fermentation of microorganisms in the presence of gelling materials, create hierarchical porous composites with unexpected potential applications in the fields of bioscaffold, fuel cells, thermal exchangers, and water filtration or oil absorption.16 Thus, combining features of such bioinspired structures with artificial materials can lead to design innovative fabrication methodologies of nanostructures.

Previous studies showed the ability of some bacteria to grow at the water-oil interface taking nutrients from the water and carbon sources from the oil phase.17 Yeast is a cellular factory and is naturally capable of transforming simple molecules, taken from its nutrition, such as sugars, and synthesizing new elements at a mild temperature. Yeast cells are widely used in industrial and biological processes.18,19 Saccharomyces cerevisiae was used for centuries in fermentation processes in wine and bread making. When sugar is added to water in the presence of the yeast, the fermentation is activated and the $\mathrm{CO} 2$ bubbles are produced as byproduct and expelled through the reaction solution. In a previous work the flotation of graphene oxide was induced in carbonated water and it was shown that graphene sheets were first captured by the rising $\mathrm{CO} 2$ bubbles and then transported to the water surface.4 Thus, nanomaterials which are processable in aqueous media were expected to selfassemble at the water/CO2 interface to form a new film that retracts and selfassembles at the interface when the $\mathrm{CO} 2$ is drained at liquid interface.

In this article we exploit the utilization of bread fermentation to prepare bionic composites with intractable polymers, presenting here the PEEK polymer as case study. Highly stable emulsions of PEEK powder results in a controlled interaction with different nanophases stabilized at oil-water interface by bubbling produced by microorganism nutrition process (i.e., baker's yeast fermentation). With this method, the microstructure and the mechanical properties of the nanocomposites can be tailored by changing the composition of the stabilized nanophase. Finally sintering of such hybrid emulsions enable the preparation of polymeric foams from a variety of polymeric particles including polymers intractable by solvent casting. Porous nanocomposites with improved mechanical strength and thermal insulating properties in the absence of any solvent and chemical reaction were obtained.

\section{Experimental details}

Graphene nanoplatelets (GNPs) were kindly supplied by NANESA (G3Nan average thickness of 9 $\mathrm{nm} \sim 25$ layers). Carbon nanotubes (NC 7000) were supplied by Nanocyl and their structure was confirmed by transmission electron microscopy having an average diameter $9.5 \mathrm{~nm}$ and an average length $1.5 \mu \mathrm{m}$. Poly- (etheretherketone) powder (PEEK, 200UFP10 VESTAKEEP) with particle diameter of $20-50 \mu \mathrm{m}$, specific density of $1.3 \mathrm{~g} / \mathrm{cm} 3$, and melting temperature of $340{ }^{\circ} \mathrm{C}$ was supplied by Evonik. Commercial grade Shell Helix Ultra oil was used for the experiment (density at $15^{\circ} \mathrm{C} 840 \mathrm{~kg} / \mathrm{m3}$, kinematic viscosity at $40^{\circ} \mathrm{C} 79.10 \mathrm{cSt}$ ASTM D445).

The oil was added to water leading to an instantaneous liquid separation interface $(2 \mathrm{~mL}$ of water and $8 \mathrm{~mL}$ of oil, respectively). In another beaker, $40 \mathrm{mg}$ of GNPs(CNTs) were dispersed for $3 \mathrm{~h}$ at room temperature in $40 \mathrm{~mL}$ of water using a sonication bath. Separately, a concentrated PEEK powder water suspension ( $26 \%$ vol) was prepared by vigorous stirring. Saccharomyces cerevisiae based commercial baker's yeast extract was used as the medium for fermentation. The yeast 
solution $(0.25 \mathrm{~g} / \mathrm{mL}$ in water) was added separately to the dispersions of GNPs, CNTs, and PEEK and stirred at $110 \mathrm{rpm}$ at $30^{\circ} \mathrm{C}$ for $1 \mathrm{~h}$. To start the fermentation, sugar (i.e., sucrose, $0.2 \mathrm{~g} / \mathrm{mL}$ ) was added. The dispersions were then heated at $35^{\circ} \mathrm{C}$ to allow the fermentation process. Once the fermentation was started, the dispersions were added to the water-oil system. Contact angles of the $\mathrm{CO} 2$ bubbles at oil-water interface were measured with an optical contact angle meter. Once the fermentable sugar was exhausted, PEEK based samples were extracted, shaped in aluminum molds, and sintered at $370{ }^{\circ} \mathrm{C}$ for $30 \mathrm{~min}$ to obtain nanocomposites. Field emission scanning microscopy (FESEM) was used to investigate the film morphology. The mechanical properties were measured by a universal testing machine with a $30 \mathrm{kN}$ cell at room temperature. The sample dimensions were $20 \mathrm{~mm}$ long with a square section of $4 \mathrm{~mm} \times 15 \mathrm{~mm}$. Calculation of the flexural stress 20 was made by using the following formula $\sigma=3 \mathrm{FL} /(2 \mathrm{bd} 2)$ while for the flexural strain we adopted the following relation, $\varepsilon=6 \mathrm{Dd} / \mathrm{L} 2$, where $F$ is the load, $L$ is the support span, $b$ is the width of test beam, $d$ is the thickness of tested beam, and $D$ is the maximum deflection of the center of the beam. Five samples for each composition were tested. Thermal conductivity measurements follow the "two thermometer-one heater" method using a custom built stage. For the water absorption test, the prepared samples were soaked in water at $23^{\circ} \mathrm{C}$ for $24 \mathrm{~h}$. Specimens are then removed, patted dry with a lint free cloth, and weighed. Water absorption is expressed as increase in weight percent as percent water absorption (i.e., (wet weight - dry weight/dry weight) $\times 100$ ). The prepared oil-water interfaces were characterized by laser light scattering, the images of such characterization as well as the FESEM characterization of baker's yeast cell and PEEK/CNTs sample are supplied as Supporting Information.

\section{Results and discussion}

Figure 1a-e shows the starting oil-water liquid system that was left for fermentation with neat yeast, yeast-sugar and yeast-sugar-GNPs(CNTs) dispersions, respectively. From Figure 1c it is evident that once the fermentation is activated a film of yeast cells is forming at oil-water interface. From the same figure it is clear that the ability of a large part of the yeast cells is to aggregate at the bottom of the vessel. Previous studies 21-23 demonstrated the hydrophobicity of yeast cells showing flocculent yeast cells with a high contact angle with water. Figure $1 \mathrm{f}-\mathrm{h}$ reports the experiment done with GNPs and shows that during the yeast fermentation, part of the yeast stabilizes at liquid interface trapping floating GNPs at the oil- water interface that was found stable for several days while the oil phase is no more transparent with the appearance of an emulsion that was formed. The same effect was observed with CNTs as reported in Figure 1e. Figure 1i shows that GNPs form a self-assembled floating film on the water surface without the need of surface functionalization or extra surfactant. From this figure it is evident that the rising $\mathrm{CO} 2$ bubbles are the byproduct of the fermentation process. These findings suggest that GNPs and CNTs behave as surface active molecules; they adhere to the rising $\mathrm{CO} 2$ bubbles and are trapped at the oil- water interface to minimize the surface energy.

The interaction between flocculent yeast cells and oil was investigated by light scattering measurements and light microscopy (Figure S1a). After the yeast fermentation of the oil phase is no more transparent because the light diffused (Figures $1 \mathrm{~g}$ and $\mathrm{S} 1 \mathrm{a}, \mathrm{b})$. This finding suggests that the visible light was scattered by diffused flocculent yeast cells. To support this hypothesis, the optical microscopy analysis (Figure S1c) reveals in the oil phase the aggregation of yeast cells around oil droplets, suggesting the formation of an emulsion of the oil and explaining the loss of the optical transparency. 

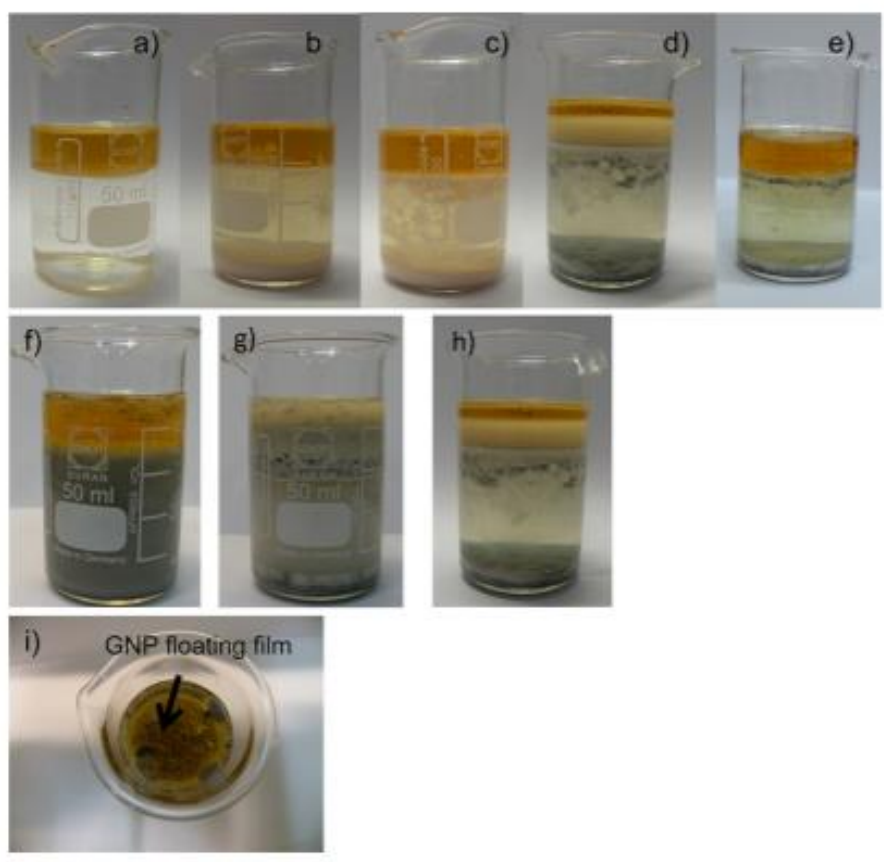

Figure 1. Images of the prepared liquid systems: (a) oil-water, (b) yeast solution in oil-water, (c) fermented yeast in oil-water, and (d) GNPs and (e) CNTs assisted yeast fermentation in oil/water after 15 days. ( $\mathrm{f}-\mathrm{h}) \mathrm{GNPs}$ assisted yeast fermentation in oil-water system at different times from preparation (from left to right): as prepared, after $24 \mathrm{~h}$ and after 15 days, respectively. (i) Top view image of GNPs assisted yeast fermentation at oil-water interface showing floating GNP based film.

Micrographs taken immediately after starting fermentation are presented in Figure 2a. Analysis of microscopy images revealed that the fermentation produces CO2 bubbles with GNPs stabilized on their surface. The bubbles move toward water-oil interface during fermentation and flocculate at the liquid interface as reported in the right panel of Figure 2a. Bubbles were found to be size sensitive to changes at liquid interface (bottom panel of Figure 2a). Different stages of the bubble migration from the fermentation bath to the oil-water interface were illustrated in Figure $2 \mathrm{~b}$. Figure $2 \mathrm{c}$ shows the contact angle and the bubble area variation during its contact with the liquid interface. When the $\mathrm{CO} 2$ bubble was released by the yeast fermentation, it was observed that it deforms once in contact with the oil-water interface leaving a thin film at the interface (Figure $2 \mathrm{~b}$ ). The images suggest that the bubble draining in the oil phase is the origin of the film release. Then the GNPs were released to the upper oil-water interface with the $\mathrm{CO} 2$ bubbles that were drained into the bulk oil phase. Wet assembly of GNPs(CNTs) at the liquid-liquid interface is driven thermodynamically. Due to the high interfacial tension of two immiscible liquid phases, entrapping the particles increases the interfacial energy and minimizes the free energy of the system. When the $\mathrm{CO} 2$ bubble is in equilibrium with its surrounding, the internal pressure $(p)$ is equal to its interfacial tension $(\gamma)$ divided by its radius $(r)$

$$
p=2 \gamma / r
$$

giving

$$
d r / r+d p / p=d \gamma / \gamma
$$

Assuming that in the bubble the mass gas and the temperature are constant

$$
p r^{3}=\text { const }
$$



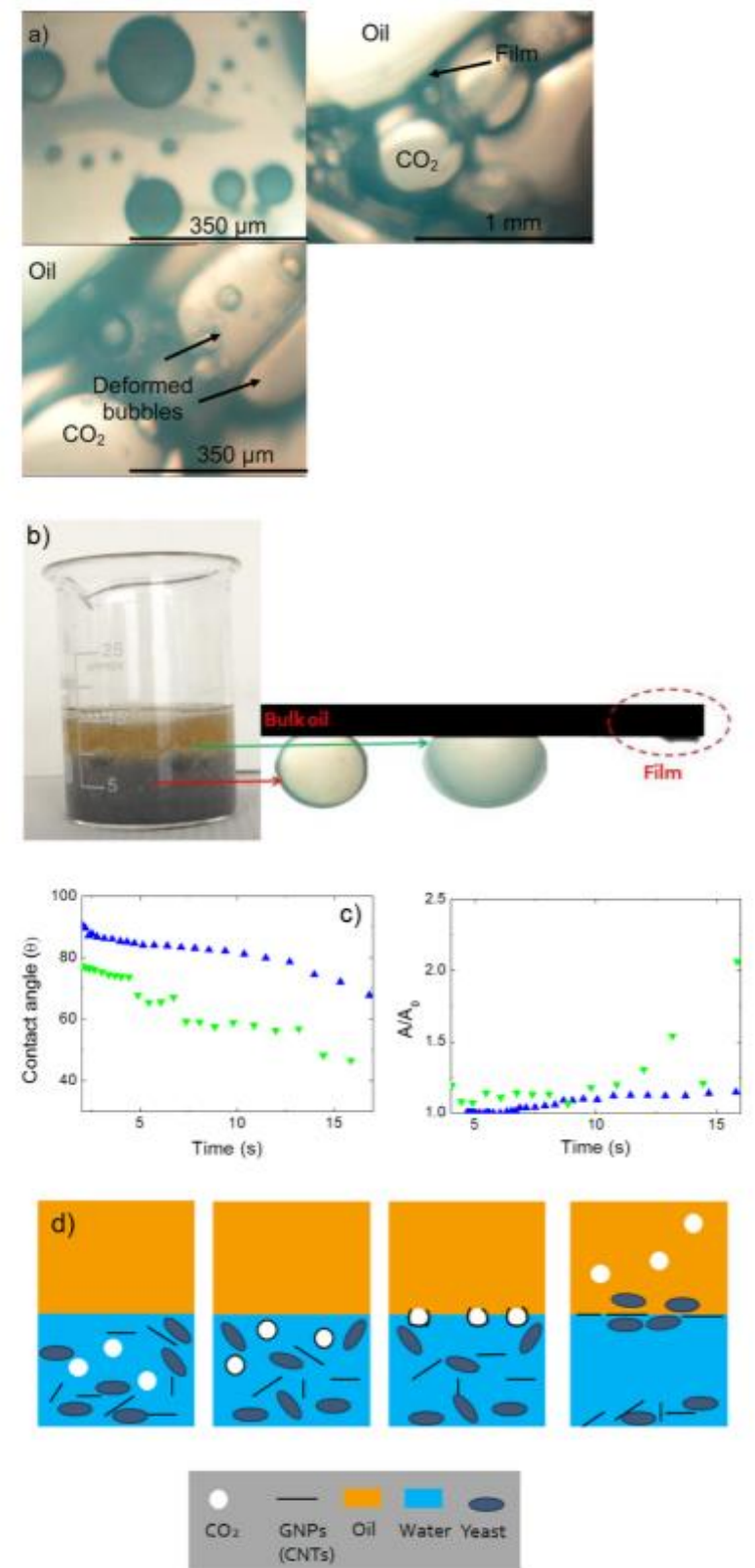

Figure 2. (a) Microscopy images of $\mathrm{CO}_{2}$ bubbles in water phase (left panel) and at oil-water interface (right panel). The bottom panel shows the optical image of deformed bubble while the film is receding at the interface. (b) Optical image of oil-water interface during fermentation (left panel) and different stages of the draining process at oil-water interface of $\mathrm{CO}_{2}$ bubble in a solution containing GNPs (right panel). (c) Contact angle and area bubble variation as a function of time for GNPs (blue triangles) and CNTs (green triangles). (d) Schematic illustration of the assembly process of the GNPs (CNTs): $\mathrm{CO}_{2}$ bubbles were released in the water phase as byproduct of the fermentation process (first panel) and a protective GNP(CNTs) coating was formed (second panel). $\mathrm{CO}_{2}$ bubbles diffuse to the oilwater interface and penetrate the oil phase (third panel); the flexible nature of the GNPs(CNTs) enabled them to orient parallel to the oil/ water interface and self-assemble (fourth panel). 
The interfacial tension of the bubble shell in water can be estimated as yw + $\sigma G N P s(C N T s) t G N P s(C N T s)$ where $y w$ is the water surface tension $\left(71 \mathrm{mN} / \mathrm{m}\right.$ at $\left.25{ }^{\circ} \mathrm{C}\right), 24$ $\sigma G N P s(C N T s)$ is the film stress, and tGNPs(CNTs) is the thickness of the retracting film once the bubble comes in contact with water-oil interface (see Figure $2 \mathrm{~b}$ ). When the bubble was drained in the bulk oil phase, the interfacial tension can be assumed as that of uncoated CO2 bubble in oil (i.e., yoil $\sim 50 \mathrm{mN} / \mathrm{m}$ ).24,25 Thus, the bubble radius deformation can be considered directly proportional to stress in the coating times its thickness ("interfacial tension"). The interfacial tension may be calculated directly from an analysis of the geometry in the deformed bubble. Assuming a GNPs(CNTs) film thickness (tGNP or tCNT) of about $8 \mathrm{~nm}(9 \mathrm{~nm})$ for the yeast based bionic composites, 15,26 we found a value for the GNPs(CNTs) film a stress value of about 30.31 $\mathrm{MPa}(26.94 \mathrm{MPa})$, respectively.

The overall schematic illustration of this process was shown in Figure 2d. When the $\mathrm{CO} 2$ bubble was released in the water phase, the GNPs(CNTs) tend to form a coating around the $\mathrm{CO} 2$ bubble; once the $\mathrm{CO} 2$ bubble is in contact with the bulk oil, it starts to merge into the bulk oil with the external GNP(CNTs) coating that retracts and assembles at the liquid interface.

Field emission scanning microscopy was used to investigate the surface of the interface once extracted from the beaker through liquid filtration. Figure 3a shows that yeasts reproduce asexually through a process called "budding ", in which a "mother" cell grows a "daughter" cell that separates to become a fully independent yeast. Figure $3 a$ also shows that the film consists of GNP sheets trapped in the yeast phase. In our previous study 15 the viability of the yeast cells after incubation with GNPs was preliminary assessed. In particular we took inspiration from yeast fermentation to couple graphene on cell wall. In turn, this process led to the development of hierarchical and selfhealing materials that could sense damage and repair it. In this regard, Kempaiah et al.19 reported that the coupling of the high modulus graphene sheets with the cells increases their stability to osmotic stresses showing a lag phase of few hours, after which they proliferate rapidly.
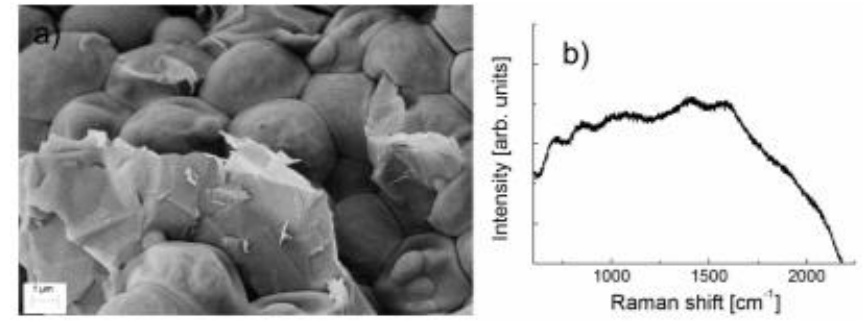

Figure 3. (a) FESEM image of film extracted after yeast fermentation showing GNPs trapped between yeast cells. (b) Raman spectrum of the sample reported in the panel (a).

Figure $3 b$ shows the Raman spectrum of such film. The main features in the Raman spectrum are the $G$ and $D$ bands. The $G$ band, standing at around $1580 \mathrm{~cm}-1$, corresponds to in-plane carbonatom stretching vibrations.27 The position of the $D$ band is excitation-energy dependent and occurs at around $1329 \mathrm{~cm}-1$. The $D$ band is activated by the presence of defects.27 From our measurements we observe a high intensity of the $D$ peak, as compared to the $G$ peak; such result is consistent with a layered and defected structures.

The utilization of solid particles as stabilizing agents of emulsions is a consolidated practice known as Pickering emulsions.28-32 Starting from this point, we could adopt the above strategy for fabrication of PEEK nanocomposites where PEEK particles work as the surfactant. Adsorption of hydrophobic particles at liquid-liquid interface is in fact originated from reduction of free energy of the system expressed as $\Delta \mu=\mathrm{A}(\mathrm{\gamma p} / \mathrm{w}-\mathrm{\gamma p} / \mathrm{o}-\mathrm{\gamma o} / \mathrm{w})$, where $\mathrm{A}$ is the surface area of the particles and $\mathrm{yp} / \mathrm{W}$ and $\mathrm{yp} / \mathrm{O}$ are the surface tension of the particle-water and the particle-oil phase, respectively. Therefore, the trapping of the polymeric particles at the interface is energetically favorable when $\mathrm{yo} / \mathrm{w}$ is higher than the difference of $\mathrm{yp} / \mathrm{w}$ and $\mathrm{yp} / \mathrm{o}$. Based on the available data for 
the surface energy of PEEK, water and oil ( $\mathrm{\gamma o} / \mathrm{w}=14.26 \mathrm{mN} / \mathrm{m}, \mathrm{\gamma p} / \mathrm{w}=22 \mathrm{mN} / \mathrm{m}$, and $\mathrm{\gamma p} / \mathrm{o}=16.83$ $\mathrm{mN} / \mathrm{m33}, 34$ ), the interfacial self-assembly of PEEK particles at the liquid-liquid interface is favored. The high stability achieved by the presence of nanoparticles allows their stabilization at the liquid interface (Figure 4a) and spanning throughout the continuous phase allowed for drying and sintering of the wet PEEK-based interface directly into nanocomposites, as shown in Figure $4 \mathrm{~b}-\mathrm{d}$. Microstructures of neat PEEK and its nanocomposites from stabilized particles are shown in Figure 5. Sintered GNPs/ PEEK structures showed pore sizes in the range from 2 to $5 \mu \mathrm{m}$ (Figure $5 \mathrm{~b}$ ). Regarding the possibility to control the number and size of the pores, it is known that during microorganism nutrition process the byproduct $\mathrm{CO} 2$ is produced from the energy gained by the yeast cell during sugar conversion. Thus, the pore density depends on the amount of nutrition (i.e., sugar) available for the cells and thus the $\mathrm{CO} 2$ expelled, while the pore size is controlled by the variation of interface energy of $\mathrm{CO} 2$ bubbles at oil-water interface as reported elsewhere.24

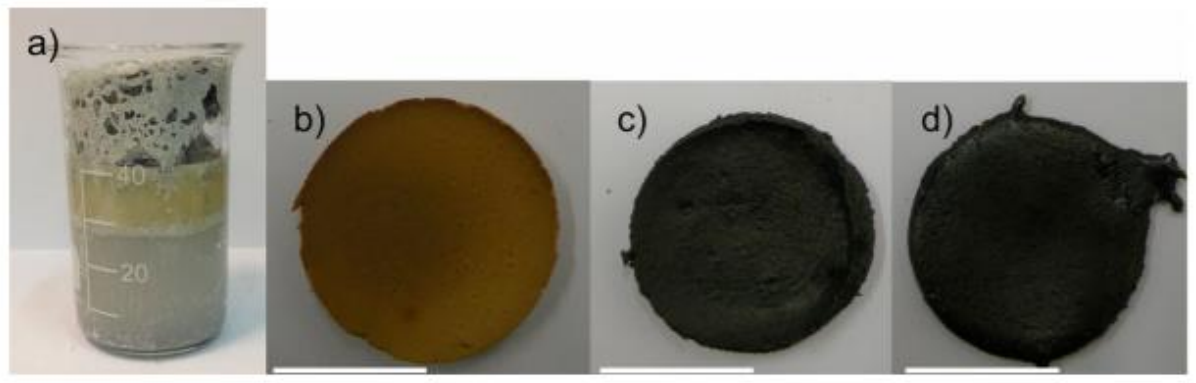

Figure 4. (a) Optical microscope image of oil-water interface prepared with PEEK particles and GNPs. Samples obtained by sintering of (b) PEEK, (c) PEEK/GNPs, and (d) PEEK/CNTs stabilized oil-water interfaces. The scale bars indicate $2.5 \mathrm{~cm}$.

Sintering of PEEK containing CNTs shows a more compact structure (Figure $5 \mathrm{c}$ ). The morphology reported in Figure $5 \mathrm{c}$ is ascribed to the high thermal conductivity of the carbon nanotubes that facilitates at some extent the complete melting of PEEK particle. In order to investigate the effect of microstructure to the heat transfer through the samples, thermal conductivity of the prepared samples with different microstructures was measured and reported in Figure $5 \mathrm{~d}$.

In a porous structure the contribution to the thermal conduction comes from solid and gas components with the thermal conductivity of the gas phase that is smaller than that of the solid one. Thus, the heat transfer is related to the porosity according to this simple model that rescales with the porosity as $\mathrm{K}=(1-(1+\alpha) f) K 0$ where $\mathrm{K}$ is the effective thermal conductivity, $\mathrm{K} 0$ is the thermal conductivity of the continuous dense matrix, $\alpha$ is a correction factor that takes into account the bulk and shear modulus of the dense matrix, and $f$ is the sample porosity (see Table 1).35 According to this relationship and assuming for a PEEK sample without pores a thermal conductivity value of $0.32 \mathrm{~W} / \mathrm{mK}$, from the data of porosity reported in Table 1 we should obtain for the thermal conductivity of our porous PEEK a value of about $0.24 \mathrm{~W} / \mathrm{mK}$ that is in good agreement with that reported in Figure 5d.

Due to the ultrahigh thermal conductivity of graphene, graphene-based materials are expected to be good thermal conductors. However, recently extremely low values of the thermal conductivity $\mathrm{K}$ of porous and light graphene and graphene-carbon nanotube hybrid aerogels was found.36,37 The proposed mechanism of this low $\mathrm{K}$ value revealed that the dominant interface thermal contact resistance of highly disordered and amorphous structure of graphene contributed to the low thermal conductivity.

Figure $5 \mathrm{~d}$ confirms also that the larger porosity shown in Figure $5 b$ contributes to thermal resistance of the GNP-based composite. In general, the obtained results indicate that the thermal conductivity of the composites is quite low. Considering that for layered WSe2 crystals and for carbon foams values of $0.05 \mathrm{~W} / \mathrm{mK}$ and $0.10-0.30 \mathrm{~W} / \mathrm{mK}$ at room temperature were obtained respectively,38,39 our findings pave the way of the utilization of such nanocomposites as thermal insulation materials. 
Figure 5e shows the mechanical characteristics obtained by three point bending test of neat PEEK and its nanocomposites. Assuming for neat PEEK without pores a density of $1.3 \times 103 \mathrm{~kg} / \mathrm{m} 3$ with a flexural strength of $70 \mathrm{MPa}, 40$ a flexural strength normalized by the density (i.e., specific strength) of about $53.80 \mathrm{kPa} /(\mathrm{kg} / \mathrm{m} 3)$ was obtained. This value, that is about 3 times higher than that observed for our neat PEEK, was expected due to the pore stress concentration. From this result it is evident that the flexural strength strongly depends on the pore density. The strength, toughness, and the deformation at break values obtained for PEEK/CNTs sample are higher than the respective data obtained for porous PEEK. The relatively high strength and toughness observed for the PEEK/GNPs sample with irregular pore size and disordered pore distribution could be related to the high moduli of GNP particles, and to the fact that the GNPs makes the pores stiffer by interconnection as reported in Figure 5b. It should be noted that the specific strength gap between our PEEK and the bulk PEEK reduced from a factor of three to a factor of two when GNPs(CNTs) were added indicating that the addition of such nanophases mitigates the presence of the pores.
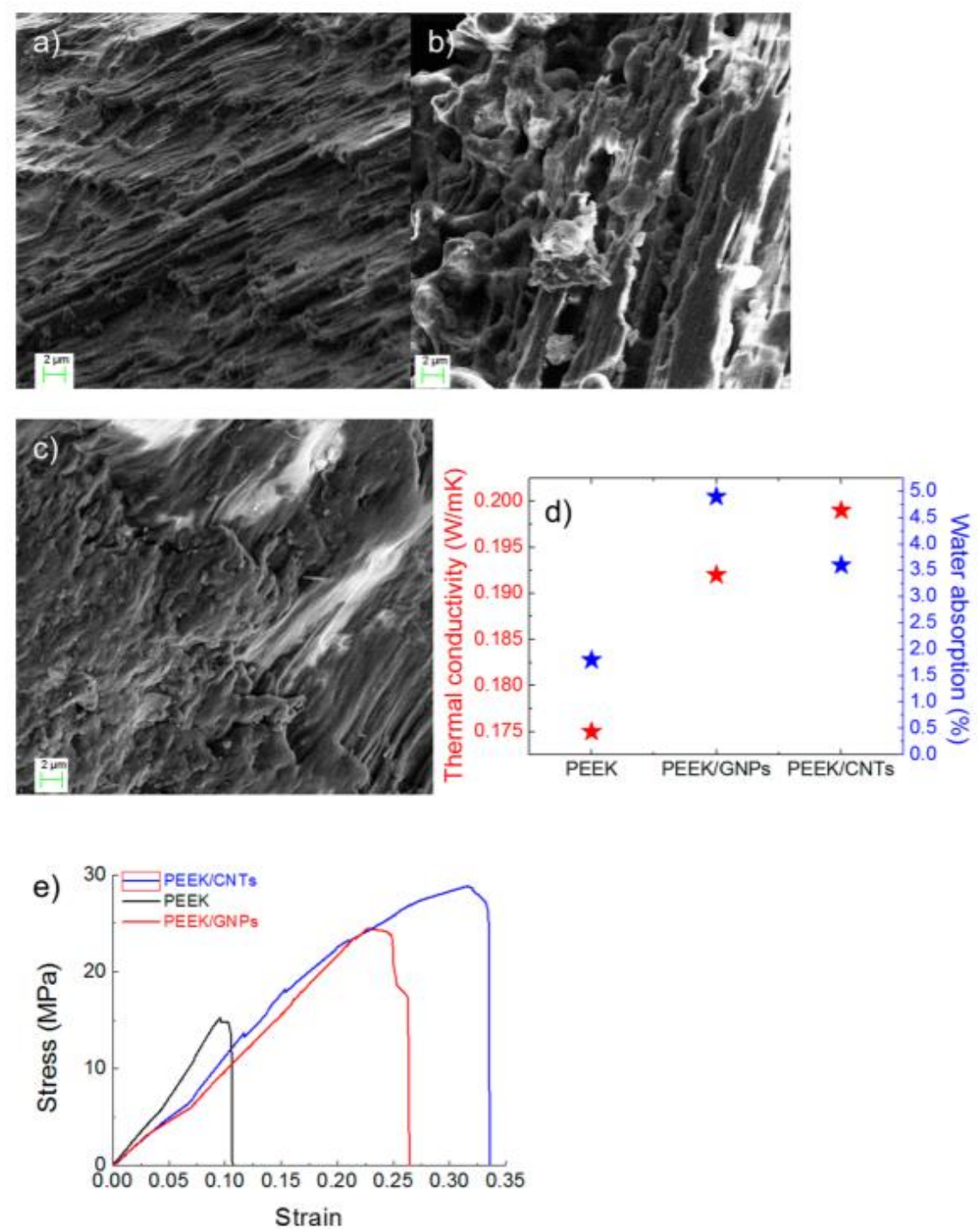

Figure 5. Microstructures of (a) PEEK, (b) PEEK/GNPs, and (c) PEEK/CNTs sintered nanocomposites. (d) Thermal conductivity, water absorption, and (e) stress-strain curves of neat PEEK and PEEK based nanocomposites. 
Table 1. Mechanical Properties of PEEK and its Composites ${ }^{a}$

\begin{tabular}{|c|c|c|c|c|c|c|}
\hline samples & $\begin{array}{c}\text { density } \\
10^{3}\left(\mathrm{~kg} / \mathrm{m}^{3}\right)\end{array}$ & $\begin{array}{l}\text { porosity } \\
(\%)\end{array}$ & $\begin{array}{l}\text { specific strength } \\
\mathrm{kPa} /\left(\mathrm{kg} / \mathrm{m}^{3}\right)\end{array}$ & $\begin{array}{l}\text { Young modulus/density } \\
\qquad \mathrm{ka} /\left(\mathrm{kg} / \mathrm{m}^{3}\right)\end{array}$ & $\begin{array}{l}\text { toughness/density } \\
\qquad \mathrm{kPa} /\left(\mathrm{kg} / \mathrm{m}^{3}\right)\end{array}$ & $\begin{array}{c}\text { deformation at break } \\
(\%)\end{array}$ \\
\hline PEEK & 1.00 & 24 & 15.04 & 155 & 0.83 & 10.2 \\
\hline $\begin{array}{l}\text { PEEK/ } \\
\text { CNTs }\end{array}$ & 1.04 & 20 & 26.92 & 100 & 5.79 & 31.7 \\
\hline $\begin{array}{l}\text { PEEK/ } \\
\text { GNPs }\end{array}$ & 0.87 & 34 & 27.58 & 129 & 3.46 & 24.8 \\
\hline
\end{tabular}

\section{Conclusions}

Inspired by the bubbling production, our study provides a potential scalable route for the production of nanocomposites. The main objective of this work was developing a simple and versatile approach to produce porous nanocomposites of various nanophase compositions. The variation of the traditional Pickering emulsion method has allowed tailoring of the microstructure and properties of nanocomposites foams in a single step process. In this regard, the fermentation process of baker's yeast was found to be a simple and versatile approach for the formation of bubbles that once contacting with the oil- water interface retract promoting the assembly of thin films of graphene nanoplatelets or carbon nanotubes at the interface. We extend this approach as a general route to assembe intractable polymers; we presented as a case study the adsorption of PEEK particles at this modified oil-water interface, allowing their stabilization and further sintering into nanocomposites with porous structures, thermal insulating properties, and enhanced mechanical characteristics. These results present a new method for the deposition control of nanomaterials and fabrication of porous nanocomposites, that may be used in many applications, as low-weight structural components, shock absorbers, thermal insulating materials for aerospace components, and robust scaffolds for tissue engineering.

\section{Author Information}

\section{Corresponding Authors}

*Tel: +39 0744 492924; E-mail: luca.valentini@unipg.it.

*Tel: +39 0461 282525; E-mail: nicola.pugno@unitn.it.

\section{Author Contributions}

L.V. and N.M.P. had the idea. L.V. designed and supervised the experimental research analyzing the experimental results. N.M.P. developed the model of the bubble and rationalized the mechanical results. S.B.B. prepared the samples and performed the characterizations. All authors have revised and given their approval to the final version of the manuscript.

\section{Funding}

N.M.P. is supported by the European Research Council (ERC StG Ideas 2011 BIHSNAM n. 279985, ERC PoC 2015 SILKENE nr. 693670), by the European Commission under the Graphene Flagship (WP14 Polymer Composites, no. 696656).

\section{Notes}

The authors declare no competing financial interest. 


\section{Acknowledgements}

Prof. Luigi Torre (University of Perugia-Italy) is acknowledged for hosting S.B.B. at the Materials Science and Technoloty labs. Prof. Bruna Bertucci and Dr. Lorenzo Mussolin (University of PerugiaItaly) are kindly acknowledged for the optical characterization of the oil/water systems. Dr. Manoj Tripathi (Fondazione Bruno Kessler-Trento) is acknowledged for Raman characterization.

\section{References}

(1) Huang, Y.; Duan, X.; Wei, Q.; Lieber, C. M. Directed Assembly of One-Dimensional Nanostructures into Functional Networks. Science 2001, 291, 630-633.

(2) Mirkin, C. A. Programming the Assembly of Two- and ThreeDimensional Architectures with DNA and Nanoscale Inorganic Building Blocks. Inorg. Chem. 2000, 39, 2258-2272.

(3) Murray, C. B.; Kagan, C. R.; Bawendi, M. G. Self-Organization of CdSe Nanocrystallites into Three-Dimensional Quantum Dot Superlattices. Science 1995, 270, 1335-1338.

(4) Kim, J.; Cote, L. J.; Kim, F.; Yuan, W.; Shull, K. R.; Huang, J. Graphene Oxide Sheets at Interfaces. J. Am. Chem. Soc. 2010, 132, 8180-8186.

(5) Tang, Z.; Zhuang, J.; Wang, X. Exfoliation of Graphene from Graphite and Their Self-Assembly at the Oil - Water Interface. Langmuir 2010, 26, 9045-9049.

(6) Fang, S.; Chen, T.; Wang, R.; Xiong, Y.; Chen, B.; Duan, M. Assembly of Graphene Oxide at the Crude Oil/Water Interface: A New Approach to Efficient Demulsification. Energy Fuels 2016, 30, 3355-3364.

(7) Zhang, Y.; Shen, Y.; Kuehner, D.; Wu, S.; Su, Z.; Ye, S.; Niu, L. Directing Single-Walled Carbon Nanotubes to Self-Assemble at Water/Oil Interfaces and Facilitate Electron Transfer. Chem. Commun. 2008, 4273-4275.

(8) Duan, H.; Wang, D.; Kurth, D. G.; Mohwald, H. Directing SelfAssembly of Nanoparticles at Water/Oil Interfaces. Angew. Chem., Int. Ed. 2004, 43, 5639-5642.

(9) Lin, Y.; Skaff, H.; Emrick, T.; Dinsmore, A. D.; Russel, T. P. Nanoparticle Assembly and Transport at Liquid-Liquid Interfaces. Science 2003, 299, 226-229.

(10) Croll, L. M.; Stö ver, H. D. H. Formation Of Tectocapsules by Assembly and Cross-Linking of Poly(Divinylbenzene-Alt-Maleic Anhydride) Spheres at the Oil-Water Interface. Langmuir 2003, 19, 5918-5922.

(11) Akartuna, I.; Tervoort, E.; Wong, J. C. H.; Studart, A. R.; Gauckler, L. J. Macroporous Polymers from Particle-Stabilized Emulsions. Polymer 2009, 50, 3645-3651.

(12) Newman, D. K. Microbiology - How Bacteria Respire Minerals. Science 2001, 292, 1312-1313.

(13) Lowenstam, H. A. Minerals Formed by Organisms. Science 1981, 211, 1126-1131.

(14) Fortin, D. What Biogenic Minerals Tell Us. Science 2004, 303, 1618-1619.

(15) Valentini, L.; Bittolo Bon, S.; Signetti, S.; Pugno, N. M. 142. Graphene Based Bionic Composites with Multifunctional and Repairing Properties. ACS Appl. Mater. Interfaces 2016, 8, 7607-7612.

(16) Zhang, Q.; Chen, B.; Tao, L.; Yan, M.; Chen, L.; Wei, Y. Microorganism Inspired Hydrogels: Hierarchical Super/Macro-Porous Structure, Rapid Swelling Rate and High Adsorption. RSC Adv. 2014, 4, 32475-32481. 
(17) Torsvik, T.; Gilje, E.; Sunde, E. Aerobic Microbial Enhanced Oil Recovery, 5th International Conference on Microbial Enhanced Oil Recovery, Dallas TX, USA, Sep 111995.

(18) Chen, Y.; Daviet, L.; Schalk, M.; Siewers, V.; Nielsen, J. Establishing a Platform Cell Factory Through Engineering of Yeast Acetyl-CoA Metabolism. Metab. Eng. 2013, 15, 48-54.

(19) Kempaiah, R.; Salgado, S.; Chung, W. L.; Maheshwari, V. Graphene as Membrane for Encapsulation of Yeast Cells: Protective and Electrically Conducting. Chem. Commun. 2011, 47, 11480-11482.

(20) Standard Test Method for Flexural Properties of Polymer Matrix Composite Materials; ASTM, 2007, D 7246M-2007.

(21) Everaert, E. P. J. M.; van der Mei, H. C.; Busscher, H. J. Adhesion of Yeasts and Bacteria to Fluoro-Alkylsiloxane Layers Chemisorbed on Silicone Rubber. Colloids Surf., B 1998, 10, 179-190.

(22) Smit, G.; Straver, M. H.; Lugtenberg, B. J.; Kijne, J. W. Flocculence of Saccharomyces Cerevisiae Cells Is Induced by Nutrient Limitation with Cell Surface Hydrophobicity as a Major Determinant. Appl. Environ. Microbiol. 1992, 58, 3709-3714.

(23) Straver, M. H.; Aar, P. C. V. D.; Smit, G.; Kijne, J. W. Determinants of Flocculence of Brewer's Yeast During Fermentation in Wort. Yeast 1993, 9, 527-532.

(24) Sun, C.-Y.; Chen, G.-J. Measurement of Interfacial Tension for the CO2 Injected Crude Oil + Reservoir Water System. J. Chem. Eng. Data 2005, 50, 936-938.

(25) da Rocha, S.R. P.; Harrison, K. L.; Johnston, K. P. Effect of Surfactants on the Interfacial Tension and Emulsion Formation Between Water and Carbon Dioxide. Langmuir 1999, 15, 419-428.

(26) Valentini, L.; Bittolo Bon, S.; Tripathi, M.; lacob, E.; Signetti, S.; Pugno, N. M. Fermentation Based Carbon Nanotube Multifunctional Bionic Composites. Sci. Rep. 2016, 6, 27031.

(27) Ferrari, A. C.; Meyer, J. C.; Scardaci, V.; Casiraghi, C.; Lazzeri, M.; Mauri, F.; Piscanec, S.; Jiang, D.; Novoselov, K. S.; Roth, S.; Geim, A. K. Raman Spectrum of Graphene and Graphene Layers. Phys. Rev. Lett. 2006, 97, 187401.

(28) Wang, T.; Colver, P. J.; Bon, S. A. F.; Keddie, J. L. Soft Polymer and Nano-Clay Supracolloidal Particles in Adhesives: Synergistic Effects on Mechanical Properties. Soft Matter 2009, 5, 3842-3849.

(29) San Miguel, A.; Scrimgeour, J.; Curtis, J. E.; Behrens, S. H. Smart Colloidosomes with a Dissolution Trigger. Soft Matter 2010, 6, 3163-3166.

(30) Wang, D.; Duan, H.; Mohwald, H. The Water/Oil Interface: the Emerging Horizon for SelfAssembly of Nanoparticles. Soft Matter 2005, 1, 412-416.

(31) Boker, A.; He, J.; Emrick, T.; Russell, T. P. Self-assembly of Nanoparticles at Interfaces. Soft Matter 2007, 3, 1231-1238.

(32) Dinsmore, A. D.; Hsu, M. F.; Nikolaides, M. G.; Marquez, M.; Bausch, A. R.; Weitz, D. A. Colloidosomes: Selectively Permeable Capsules Composed of Colloidal Particles. Science 2002, 298, 1006- 1009.

(33) Loy, X. Z. K.; Sinha, S. K. Lubrication of Polyether Ether Ketone (PEEK) Surface by Liquid Ultrathin Films for High Wear Durability. Wear 2012, 296, 681-692.

(34) Than, P.; Preziosi, L.; Josephl, D. D.; Arney, M. Interfacial Tension with Spinning Rod Tensiometer. J. Colloid Interface Sci. 1988, 124, 552-559. 
(35) DeVera, A. L.; Strieder, W. Upper and Lower Bounds on the Thermal Conductivity of a Random, Two-Phase Material. J. Phys. Chem. 1977, 81, 1783-1790.

(36) Fan, Z.; Zhi, D.; Tng, Y.; Xue, C.; Lim, T.; Liu, P.; Nguyen, S.- T.; Xiao, P.; Marconnet, A.; Lim, C.Y.-H.; Duong, H. M. Thermal and Electrical Properties of Graphene/Carbon Nanotube Aerogels. Colloids Surf., A 2014, 445, 48-53.

(37) Xie, Y.; Xu, S.; Xu, Z.; Wu, H.; Deng, C.; Wang, X. Interfacemediated Extremely Low Thermal Conductivity of Graphene Aerogel. Carbon 2016, 98, 381-390.

(38) Chiritescu, C.; Cahill, D. G.; Nguyen, N.; Johnson, D.; Bodapati, A.; Keblinski, P.; Zschack, P. Ultralow Thermal Conductivity in Disordered, Layered WSe2 Crystals. Science 2007, 315, 351-353.

(39) Yuan, Y.; Ding, Y.; Wang, C.; Xu, F.; Lin, Z.; Qin, Y.; Li, Y.; Yang, M.; He, X.; Peng, Q.; Li, Y. Multifunctional Stiff Carbon Foam Derived from Bread. ACS Appl. Mater. Interfaces 2016, 8, 1685216861.

(40) Rae, P. J.; Brown, E. N.; Orler, E. B. The Mechanical Properties of Poly(Ether-Ether-Ketone) (PEEK) with Emphasis on the Large Compressive Strain Response. Polymer 2007, 48, 598-615. 